\title{
Human small intestinal contractions and aboral traction forces during fasting and after feeding
}

\author{
N K Ahluwalia, D G Thompson, J Barlow, L Heggie
}

\begin{abstract}
Small intestinal intraluminal pressure activity and aboral traction forces were explored in 19 healthy volunteers using a combined manometry and traction force detecting assembly sited in the upper small intestine. Each aboral traction event was classified as being associated with either a propagating or a stationary contraction and its force measured. During phase I no contractions or traction events were seen. During phase II, traction events related to propagating contractions mean (SEM) $(2 \cdot 2$ $(0.2) / \mathrm{min})$ and to stationary contractions $(0.3(0.1) / \mathrm{min})$ generated similar force/ event $(7.5(0.9 \mathrm{~g} v 8.7(1.4) \mathrm{g}, \mathrm{p}>0.05)$. During phase III, all traction events were related to propagating contractions and generated $\mathbf{9 . 3}$ (2.4) $g$ force/event ( $p>0.05 v$ phase II). After feeding, traction events related to propagating contractions generated similar force/event to those related to stationary contractions $(5.9$ $(1.0) g v 9.3(2.7) g, p>0.05 v$ each other and $v$ fasting). No consistent pattern was seen in the temporal distribution of the traction events or in the pattern of the amplitude of the force of successive traction events.

(Gut 1994; 35: 625-630)
\end{abstract}

Recordings of human jejunal motor activity during the interdigestive and fed state show two main types of contractions. ${ }^{1}$ Those that travel at least $2 \mathrm{~cm}$ aborally are described as propagating contractions, while those that travel less than $2 \mathrm{~cm}$ are termed non-propagating ${ }^{1}$ or stationary contractions. ${ }^{2-6}$ This classification is based on the finding, ${ }^{36}$ that movement of intraluminal contents does not occur with contractions that fail to propagate more than $2 \mathrm{~cm}$ while those that do propagate $2 \mathrm{~cm}$ or further, move intraluminal contents aborally. Data on the relation between contraction type and luminal transit are inconsistent, however. Stationary contractions are increased in number after a nutrient meal compared with fasting phase II, although the number of propagating contractions remain unchanged. ${ }^{136}$ In contrast, small intestinal transit is faster after a nutrient meal than during the fasting phase II pattern. ${ }^{7}$

Figure 1: Diagram of the components of the distal end of the traction catheter assembly.

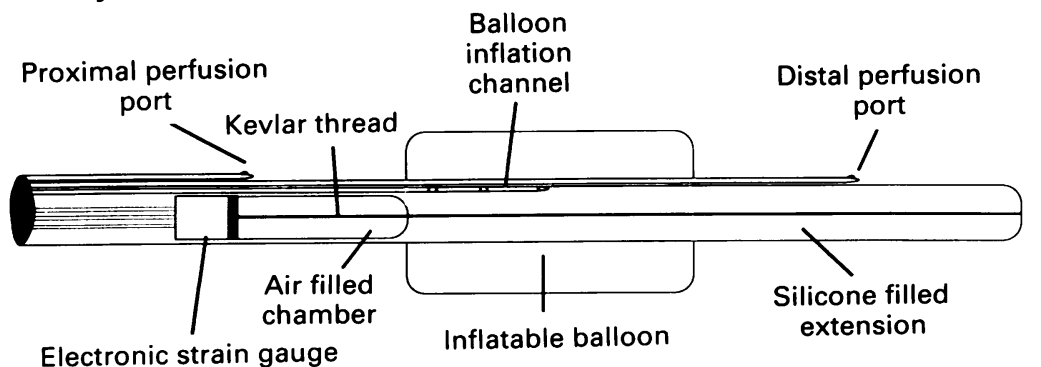

It has also been suggested that the length of propagation of contractions is reponsible for the speed of transit. ${ }^{36}$ In humans, however, the average distance travelled by a propagating contraction remains unchanged by a meal, ${ }^{1}$ so that the postprandial transit acceleration cannot be explained on the basis of propagation distance alone. Another possible explanation for the meal induced transit changes is that the propulsive forces generated by contractions are greater after a meal. So far, however, propulsive forces generated remain unexplored.

The aim of our study was to test the hypothesis that the propulsive forces generated in the small intestine would be modified by food ingestion in a manner consistent with the observed transit effects. We therefore measured the aboral forces generated within the small bowel during fasting and after a meal.

\section{Subjects}

Nineteen healthy volunteers (nine men; mean age 27 years; range 21-39) without past or current history of gastrointestinal disease participated in the study after providing informed consent.

\section{Methods}

THE TRACTION FORCE DETECTOR

This device (Fig 1), which has been previously described in detail, ${ }^{8}$ consisted of a miniature electronic strain gauge force transducer (Gaeltec Ltd, Dunvegan, Isle of Skye, UK) mounted on a catheter to which a silicone extension was attached by Kevlar threads. Because of their inelasticity, the Kevlar threads ensured that aboral traction forces acting on the extension were transferred directly to the force transducer without damping.

The force transducer was calibrated before and after each study by suspending the catheter vertically and applying known weights to the extension piece. The voltage output from the transducer amplifier was recorded on a polygraph (Grass Instruments Co, Quincy, Mass, USA) calibrated so that a $10 \mathrm{~mm}$ pen deflection was equivalent to $10 \mathrm{~g}$ force.

\section{BALLOON}

A roughly cylindrical, latex balloon, $1.5 \mathrm{~cm}$ in length (HSC4 Precision Dippings Marketing, Stover Trading Estate, Yate, UK) was attached to the silicone extension $3 \mathrm{~cm}$ proximal to the end of the catheter. During each experiment the balloon was inflated with $1.5 \mathrm{ml}$ air through a channel running through the catheter, this 
volume being chosen from the results of pilot studies because it permitted the detection of both pressure changes and aboral forces without itself inducing any distension related contractile responses.

\section{INTRALUMINAL MANOMETRY}

Intraluminal pressure activity was measured, both proximal and distal to the balloon, by attaching two polyvinyl chloride perfusion catheters (internal diameter $0.63 \mathrm{~mm}$, Portex Ltd, Hythe, Kent, UK) to the traction catheter. The perfusion port of one catheter was sited $2 \mathrm{~cm}$ proximal to the mid point of the balloon while the other was $2 \mathrm{~cm}$ distal (Fig 1). Two additional perfusion catheters with ports 7 and $17 \mathrm{~cm}$ proximal to the balloon were also attached to the catheter to aid the placement of the device in the duodenum. All the perfusion catheters were bonded to the traction catheter using tetrahydrofuran. The external diameter of the entire catheter assembly was $4.5 \mathrm{~mm}$.

Each of the four channels was perfused with degassed water at a rate of $0.5 \mathrm{ml} / \mathrm{min}$ using a low compliance pneumohydraulic perfusion system (Arndorfer Medical Specialties Inc, Greendale, Wisconsin, USA) run at a pressure of 15 pounds per square inch. A transducer (Statham P23-ID, Gould Inc, Oxnard, Ca 93030, USA) was attached to the proximal end of each of the four perfused channels, the outputs of each transducer amplifier being recorded on the polygraph, run at a paper speed of $50 \mathrm{~mm} / \mathrm{min}$ and calibrated so that a pen deflection of $10 \mathrm{~mm}$ on the chart recorder corresponded to a pressure change of $10 \mathrm{~mm} \mathrm{Hg}$. Sudden complete occlusion of each channel gave a pressure rise of $>400 \mathrm{~mm} \mathrm{Hg} / \mathrm{s}$.

To record the intraluminal pressure at the site of the balloon, the intraballoon pressure was also recorded continuously on the chart recorder using a similar Statham P23 transducer connected to the balloon inflation channel.

\section{TEST MEAL}

A mixed nutrient test meal was used for all studies (H J Heinz and Co Ltd, Cream of Chicken Soup $360 \mathrm{ml} ; 425 \mathrm{~g} ; 200 \mathrm{kcal}$; fat= $11.5 \mathrm{~g}$; protein $=5.5 \mathrm{~g}$ ). Before ingestion, the meal was warmed to a temperature of $37^{\circ} \mathrm{C}$ in a microwave oven.

\section{STUDY PROTOCOI}

The study protocol was submitted to, and approved by, the Salford District ethics committee.

All studies were conducted in the morning after an overnight fast of at least 11 hours, the subjects reclining supine with their trunk at an angle of $30^{\circ}$ to the horizontal throughout the study. The catheter assembly was first passed through the nose and the tip advanced into the stomach. The catheter was then passed through the pylorus and into the duodenum, its position being monitored by the pattern of the manometric events recorded from the perfusion channels. The identification of a typical duodenal manometric pattern from the three distal perfusion channels, and an antral pattern from the most proximal port, was taken to show that the balloon was sited in the duodenum, $12-15 \mathrm{~cm}$ beyond the pylorus. After correct placement, the catheter was tethered to the cheek with adhesive tape to prevent aboral movement, and the balloon was inflated.

A continuous recording of fasting manometric and aboral traction activity was then made for $\mathbf{6 0}$ minutes, after which the subject ingested the test meal. After a further five minutes to permit a fed pattern to develop, a 30 minute recording of postprandial activity was made.

\section{Definition of terms}

The criteria used for the classification of small intestinal contractions in this study are identical to those previously reported for humans, and are based on the assumption that the aboral propagation velocity of a contraction would be approximately $1 \mathrm{~cm} / \mathrm{s}$.

Contraction was defined as the occurrence of a pressure change either at a perfusion port or at the balloon.

Propagating contraction was defined as a contraction at the balloon that occurred between 1-2 seconds after a contraction in the proximal channel or between 1-2 seconds before a contraction in the distal channel, or both.

Stationary contraction was defined as a contraction at the balloon that did not occur either 1-2 seconds after a proximal contration or 1-2 seconds before a distal contraction. The simultaneous occurrence of pressure activity in all channels was considered to be a result of an extraintestinal artifact and was not included in data analysis.

Traction events were defined as all events recorded by the traction force detector, other than those associated with artifacts as described above. These were subdivided as being either: (a) Related to propagating contractions - that is, those that occurred synchronously with a propagating contraction; or (b) related to stationary contractions - that is, those that occurred synchronously with a stationary contraction.

Traction force - This was defined as the force in grams (g) exerted by a traction event.

\section{Data collection}

\section{CONTRACTIONS}

During fasting, the number of contractions at the proximal and distal perfusion ports and at the balloon were counted during a 30 minute segment of phase II activity (excluding the first 10 minutes after phase I) and in addition during phase III and during phase I.

During the 30 minute fed state, all contractions at the balloon and at the proximal and distal perfusion ports, were counted.

The perfusion/balloon contraction ratio was calculated as:

Proximal perfusion port contractions + distal perfusion port contractions 


\section{TRACTION EVENTS}

All traction events occurring during the study periods were identified and the force generated by each was calculated from the amplitude of the deflection on the chart recorder.

\section{TRACTION FORCE/MINUTE}

The total force generated in each period was calculated by dividing the total force by the duration of the recording period.

\section{AVERAGE TRACTION FORCE/EVENT}

The average traction force/event was calculated by dividing the total force generated by the number of events. This was calculated both for traction events related to propagating contractions and those related to stationary contractions.

\section{PATTERN OF TRACTION FORCE ACTIVITY}

To investigate in detail the temporal dispersion of the occurrence and the amplitude of successive traction events, data from three randomly selected individual studies were taken and both the time interval between successive traction events and their amplitude was noted.

\section{Statistical methods}

The distribution of the data was first tested by applying the Shapiro-Wilk test, ${ }^{9}$ which showed that all data were normally distributed. The results are therefore expressed in the text as mean (standard error of the mean).

Student's paired $t$ test ${ }^{10}$ was used for comparison of paired data. Because three comparisons of the data were made (between phases II, III, and the fed state), a p value of $<0.017$ ( 0.05 divided by the number of comparisons) was taken to show a biologically relevant difference.

The temporal dispersion of the occurrence and the amplitude of traction events was analysed by applying the method of autocorrelation. In this method, all findings in a time series are correlated successively with the same series, with a lag of $1,2,3 \ldots, x$, where $x$ is $10+V \mathrm{n}, \mathrm{n}$ being the number of findings in the series. ${ }^{11}$

The temporal relation between traction events related to propagating and those related to stationary contractions was tested by applying the non-parametric Runs test." This method assesses whether a sequence of consecutive find-

Traction data during fasting and after feeding

\begin{tabular}{|c|c|c|c|}
\hline & Phase II & Phase III & Fed state \\
\hline \multicolumn{4}{|l|}{ Traction events/min related to: } \\
\hline Propagating contractions & $\begin{array}{l}2 \cdot 2(0 \cdot 2)^{\star} \\
0 \cdot 3(0 \cdot 1)\end{array}$ & $10.5(0.6)$ & $\begin{array}{l}2 \cdot 2(0 \cdot 3)^{\star} \\
1 \cdot 1(0 \cdot 2) t\end{array}$ \\
\hline \multicolumn{4}{|c|}{ Traction force $\mathrm{g} / \mathrm{min}$ exerted by traction events related to: } \\
\hline $\begin{array}{l}\text { Propagating contractions } \\
\text { Stationary contractions }\end{array}$ & $\begin{array}{l}18 \cdot 3(2 \cdot 4)^{\star} \\
2 \cdot 5(0 \cdot 7)\end{array}$ & $98 \cdot 3(26 \cdot 9)$ & $\begin{array}{l}14 \cdot 2(2 \cdot 1)^{\star} \\
9 \cdot 4(2 \cdot 1) \dagger\end{array}$ \\
\hline \multicolumn{4}{|c|}{ Traction force g/event related to: } \\
\hline $\begin{array}{l}\text { Propagating contractions } \\
\text { Stationary contractions }\end{array}$ & $\begin{array}{l}7 \cdot 5(0 \cdot 9) \\
8 \cdot 7(1 \cdot 4)\end{array}$ & $9 \cdot 3(2 \cdot 4)$ & $\begin{array}{l}5 \cdot 9(1 \cdot 0) \\
9 \cdot 3(2 \cdot 7)\end{array}$ \\
\hline \multicolumn{4}{|l|}{ Contractions/min } \\
\hline Proximal port & $3 \cdot 7(0 \cdot 4)$ & $11 \cdot 3(0 \cdot 5)$ & $4 \cdot 8(0 \cdot 4)$ \\
\hline Balloon & $2 \cdot 5(0 \cdot 2) \ddagger$ & $10.9(0.5)$ & $3 \cdot 3(0 \cdot 3) \ddagger$ \\
\hline Distal port & $3 \cdot 5(0.3)$ & $10 \cdot 5(0 \cdot 6)$ & $5 \cdot 1(0 \cdot 5)$ \\
\hline
\end{tabular}

${ }^{\star} \mathrm{p}<0.001 v$ phase III $; \mathrm{t}<<0.01 v$ phase II; $\ddagger \mathrm{p}<0.001 v$ proximal and distal perfusion ports. Data shown as mean (SEM). ings of more than one category in a time series are randomly occurring.

\section{Results}

The Table and Figures 2-4 show the traction data during fasting and after feeding.

\section{FASTING STATE}

The characteristically irregular pattern of phase
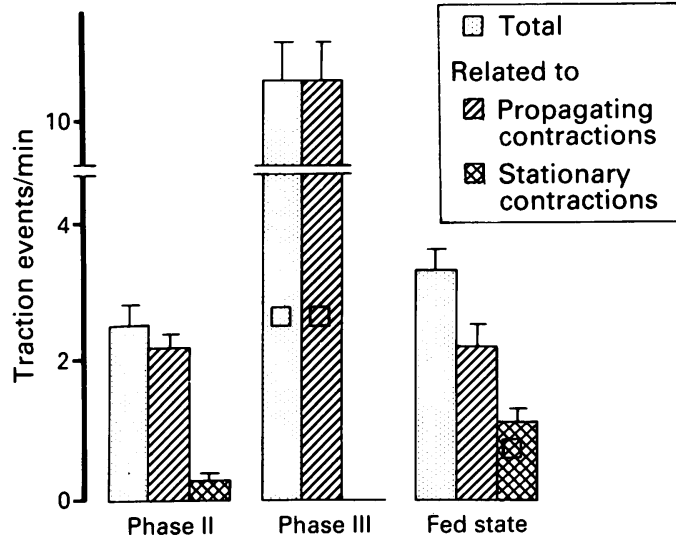

Figure 2: The frequency of traction events/min during phases $I I, I I I$, and after food is shown. Note that a greater proportion of traction events are related to stationary contractions in the fed state than during fasting.

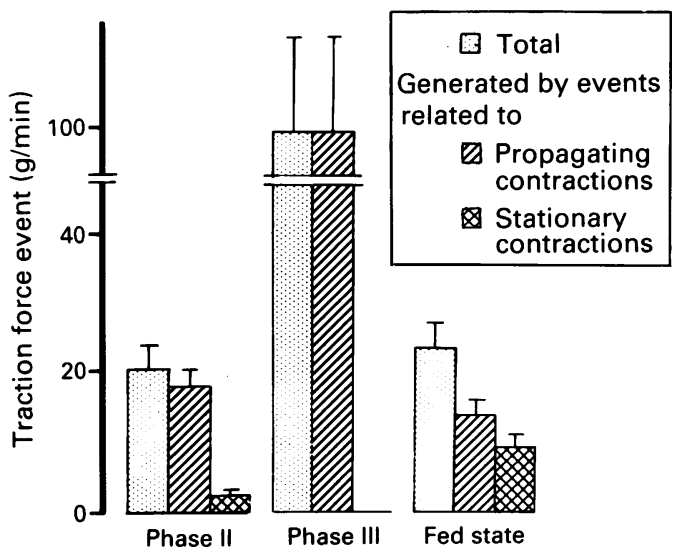

Figure 3: The traction force generated/minute is shown during phases II, III, and after food.

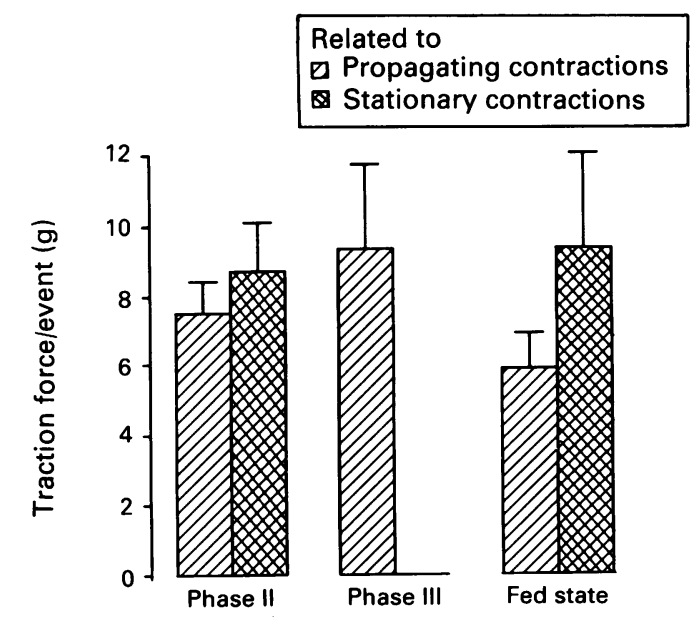

Figure 4: The traction force in g/event generated by traction events related to propagating and stationary contractions during phases II and III and after food. 
Fasting
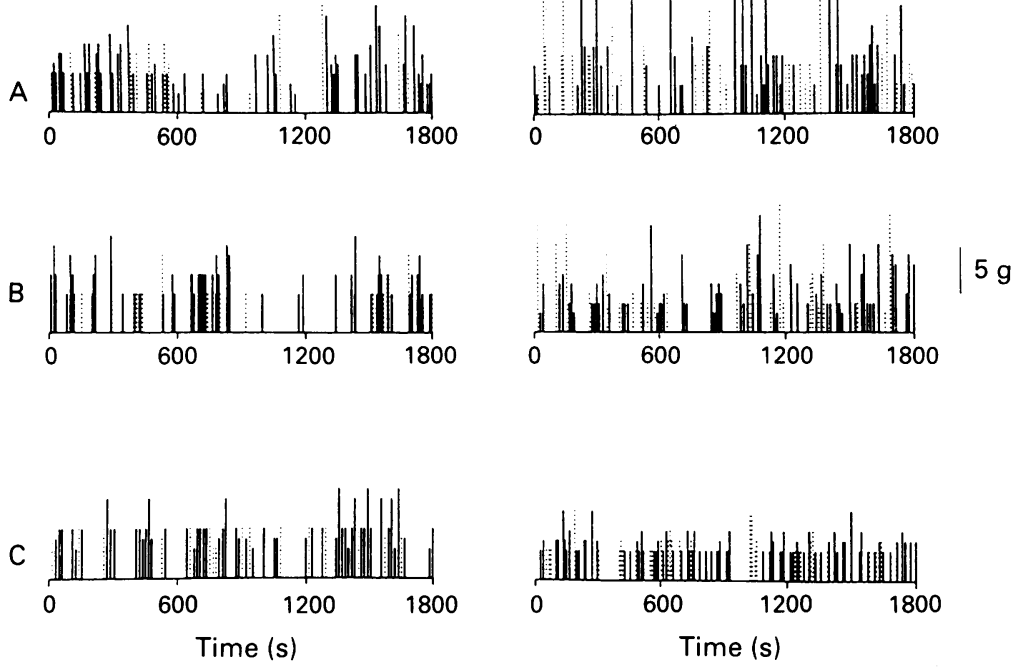

Fed
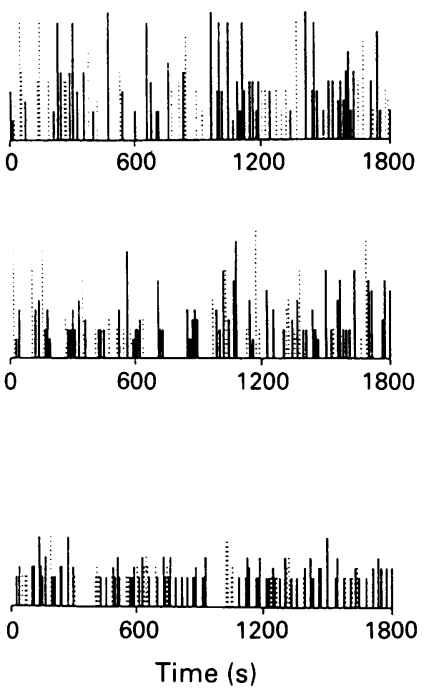

Figure 5: Randomly irregular pattern of successive traction events. The continuous lines represent traction events related to propagating contractions, while dotted lines represent traction events related to stationary contractions, in three randomly selected individual studies, $A, B, C$

II activity was seen in all 19 subjects. In seven subjects, phase III and phase I activity were also seen.

Phase I

No traction events or contractions were observed.

\section{Phase II}

Contractions - The number of contractions recorded at the proximal and distal perfusion ports were similar $(p=0.3)$, and both were consistently greater than the contractions recorded at the balloon $(p<0.001)$, with a perfusion/balloon contraction ratio of $1 \cdot 5(0 \cdot 01)$.

Traction events related to propagating contractions were greater in number and generated greater force/min than those related to stationary contractions $(87 \% v 13 \%)$ (Figs 2 and 3). The average force, however, generated by both types of traction events was similar $(p=0 \cdot 1$, Fig 4$)$. No consistent temporal dispersion pattern was seen, for either the occurrence or the amplitude of successive traction events (Fig 5). In addition the occurrence of successive traction events related to propagating contractions $v$ those related to stationary contractions were randomly irregular.

\section{Phase III}

The duration of this phase was $4 \cdot 3(0 \cdot 6)$ minutes.

Contractions - The number of contractions recorded at the proximal and distal perfusion ports and at the balloon were similar $(p>0.07$ for all comparisons) with a perfusion/balloon contraction ratio of $1.05(0.02)(\mathrm{p}<0.01 v$ phase II).

Traction events - All traction events were related to propagating contractions and although the force generated/minute was greater than phase II (Figs 2 and 3), the force generated/ traction event was similar in both phases (Fig 4).

FED STATE

\section{Contractions}

The number of contractions recorded at the proximal and distal perfusion ports were similar $(p=0.6)$, and both were greater than the number of contractions recorded at the balloon $(\mathrm{p}<0.001)$, with a perfusion/balloon contraction ratio similar to that of phase II $(1.52(0.04)$, $\mathrm{p}=0 \cdot 72$ ).

\section{Traction events}

Traction events related to propagating contractions were similar in number to phase II, and generated similar force/event. In contrast, more traction events related to stationary contractions occurred than in phase II, $\mathrm{p}<0.001$, and greater force was thus generated/minute.

No consistent pattern was seen in either the temporal dispersion of the occurrence or the amplitude of successive traction events (Fig 5). In addition, the occurrence of traction events related to propagating contractions and stationary contractions seemed randomly irregular.

\section{Discussion}

Our study has shown that, in the human small intestine, aboral traction forces of similar magnitude are generated both during fasting and after meal ingestion in a randomly irregular pattern.

Because stationary contractions do not seem to be associated with movement of intraluminal contents, ${ }^{3612}$ it might have been predicted that they would have been associated with less traction force than propagating contractions. Our data are inconsistent with this prediction as both types of contraction were associated with aboral forces of similar magnitude.

We also found, as have others, ${ }^{136}$ that after feeding, the number of propagating contractions remains unchanged, while the number of stationary contractions increases. As it has been shown that intraluminal transit is faster after a meal compared with phase II $^{7}$ and as we have shown that similar forces are associated with stationary and propagating contractions, it is possible to speculate that the increased number of stationary contractions is responsible for transit acceleration after the meal. Against this suggestion, however, is the finding that transit is slower after a nutrient, compared with a nonnutrient, meal even though stationary contractions increase. ${ }^{36}$ It is theretore difficult to explain why a nutrient induced increase in stationary contractions does not generate greater aboral traction force and thus accelerate transit, unless the aboral forces are in some way balanced by oral forces of similar magnitude.

In considering this possibility, it is relevant to note that both during phase II and after food, the number of contractions recorded at the balloon was less than the number recorded by either of the perfusion ports, although it was expected that the number would have been similar. ${ }^{13-16}$ This discrepancy does not seem to be explained by a difference between the ability of the perfusion ports and the balloon to detect contractions, as we have shown the ratio of perfusion to 
balloon contractions varied with the phase of motility studied. The most probable explanation, therefore, seems to be orad movement of the catheter, induced by stationary contractions. Although the catheter was tethered to prevent aboral movement, it was, however, free to move in an orad direction, in response to any forces generated immediately below the balloon, which would then have been missed both by the traction detector, and by the balloon pressure transducer. The suggestion that all these 'missed' contractions were indeed stationary contractions is supported by our finding that during phase III, when all contractions were propagating, the number of contractions at the balloon were equal to the number of contractions recorded from the perfusion ports. On the assumption therefore that most of these 'missed' contractions at the balloon were associated with orad forces, it is probable that the net propulsive force associated with stationary contractions in the fed state would have been small, as the number of missed contractions equalled those recorded.

If, as seems probable, the overall effect of stationary contractions is non-propulsive and there is no contribution to transit, ${ }^{13612}$ then the transit acceleration seen after meal ingestion could be attributed to a change in propulsive force associated with propagating contractions. This does not seem probable, however, as both the number, and strength, of the traction force associated with propagating contractions were similar during fasting and after feeding. Moreover, the distance travelled by a propagating contraction is known to be similar under both conditions.

It therefore seems from our data that neither the number or the type of traction event explain the change seen previously in transit between fasting and feeding and further exploration of the problem is required, in particular study of the relation between aboral force, and factors, such as viscosity of luminal contents, intestinal wall tone, and luminal diameter, all of which could also independently influence flow and traction force.

During fasting the catheter is situated in an empty lumen whereas after feeding it is sited in a lumen containing nutrients, which could have provided a resistance to aboral movement of the device. If this was a relevant factor in our experiments, then the traction forces detected would have been lower than those actually generated by gut contractions. It remains to be determined, however, how such an effect would actually explain the discrepancies seen between force and transit. A further factor that has to be considered is that forces of the same magnitude could influence the magnitude of luminal flow differently during fasting and after feeding. For example, a decrease in wall tone and a resulting increase in luminal diameter after feeding may favour greater aboral flow for a given luminal force generated. Further studies are needed to explore these possibilities.

Our data also contribute to the debate about the comparative contributions made by phases II and III to the maintenance of fasting intraluminal clearance. ${ }^{118}$ Because all the propagat- ing contractions in a segment of fasting small intestine are associated with aboral forces of equal magnitude, the efficiency of aboral movement is probably related both to the number of propagating contractions and to the duration of that phase. Thus although there is a four to fivefold increase in the traction force generated/ minute during phase III, the greater duration of phase II (4-10 times longer than phase III) ${ }^{13-16}$ suggests that both phase II and III may be equally important for the maintenance of clearance of the fasting small intestine. It must be accepted, however, that such a speculation presumes that both the pattern of contractions and the forces generated remains similar throughout the length of the entire small bowel.

It is of further note that both the pattern and the amplitude of the traction events occurred in a randomly irregular manner, both during fasting phase II and after feeding. Although it is also known that the pattern of small intestinal contractions is randomly irregular, ${ }^{19}$ it cannot be assumed that the irregular pattern of traction events was a result of the irregular pattern of contractions. Both the ambient intraluminal pressure and traction force depend upon several independent factors, such as wall tension, wall diameter, the nature of intraluminal contents, and the pressure gradient across the sensing site ${ }^{20}$ and it is not known how these factors interact.

In conclusion; the measure of aboral traction forces in the small bowel, show the presence of a randomly irregular pattern of traction events in the human proximal small intestine, which are similar during phase II and after feeding and are independent of the type of contraction associated.

The authors wish to thank Mrs J Young for preparing the manuscript, Gaeltec Limited for construction of the traction force detector, and Ms Sally Hollis for the statistical advice. Some of the data included in this paper were presented at the Spring meeting of the British Society of Gastroenterology in 1991 (Gut 1991; 32: A591).

1 Sarna SK, Soergel KH, Harig JM, et al. Spatial and temporal patterns of human jejunal contractions. Am f Physiol 1989 257: G423-32.

2 Schemann M, Ehein H-J. Mechanical characteristics of phase II and phase III of the interdigestive migrating moto complex in dogs. Gastroenterology 1986; 91: 117-23.

3 Schemann M, Ehrlein H-J. Postprandial patterns of canine jejunal motility and transit of luminal content. Gastroenterology 1986; 90: 991-1000.

4 Siegle M-L, Ehrlein H-J. Interdigestive contractile patterns of the ileum in dogs. Am f Physiol 1987; 253: G452-60.

5 Ehrlein H-J, Schemann M, Siegle M-L. Motor patterns of small intestine determined by closely spaced extraluminal small intestine determined by closely spaced extraluminal transducers

6 Siegle M-L, Ehrlein H-J. Digestive motor patterns and transit of luminal contents in canine ileum. Am $\mathcal{f}$ Physiol 1988; 254: G552-9.

7 Kerlin P, Zinsmeister A, Phillips S. Relationship of motility to flow of contents in the human small intestine. Gastroenterology 1982; 82: 701-6.

8 Williams D, Thompson DG, Marples M, Heggie L, O'Hanrahan T, Mani V, et al. Identification of an abnorma esophageal clearance response to intraluminal distension in patients with esophagitis. Gastroenterology 1992; 103: $943-$ 53.

9 Minitab. Minitab Statistical Software, DOS microcomputer version, release 7, 1989. Minitab Inc, 3081 Enterprise version, release 7, 1989. Minitab Inc,

10 Armitage P, Berry G. Statistical methods in medical research. Oxford: Blackwell Scientific, 1987: 141-59.

Oxford: Blackwell Scientific, 1987: 141-59.
11 SPSS/PC +. SPSS/PC + V2.0 Statistical Software, 1988. SPSS Inc, 444 N Michigan Avenue, Chicago, Illinois 60611, USA.

Inc, 444 N Michigan Avenue, Chicago, Illinois 60611 ,
Ramorino MA, Colagrande C. Intestinal motility: preliminary studies with telemetry capsules and synchronized fluorocinematography. American fournal of Digestive Diseases 1964; 9:64-71.

13 Szurszewski JH. A migrating electric complex of the canine small intestine. Am f Physiol 1969; 217: 1757-63. 
14 Code CF, Marlett JA. The interdigestive myo-electrical complex of the stomach and small bowel of dogs. $\mathcal{F}$ Physiol (Lond) 1975; 246: 289-309.

15 Sarna SK. Cyclic motor activity; migrating motor complex. Gastroenterology 1985; 89: 894-913.

16 Thompson DG, Wingate DL, Archer L, Benson MJ, Green WJ, Hardy RJ. Normal patterns of human upper small bowel motor activity recorded by prolonged radiotelemetry. Gut 1980; 21: 500-6.

17 Sarr MG, Kelly KA. Patterns of movement of liquids and solids through the canine jejunum. Am $\mathcal{F}$ Physiol 1980; 239: G497-503.

18 Summers RW, Helm J, Christensen J. Intestinal propulsion in the dog. Its relation to food intake and the migratory myoelectric complex. Gastroenterology 1976; 70: 753-8.

19 Ehrlein HJ, Schmid HR, Fienle C. Characteristic motor patterns of phase II and behaviour of phase III in the fed state. Fournal of Gastrointestinal Motility 1992; 4:317-27.

20 Macagno EO, Christensen J. Fluid mechanics of the duodenum. Annual Reviews of Fluid Mechanics 1980; 12 . denum. 